archives-ouvertes

\title{
UWB channel modeling for objects evolving in impulsive environnements
}

\author{
Nourddine Azzaoui, Laurent Clavier
}

\section{To cite this version:}

Nourddine Azzaoui, Laurent Clavier. UWB channel modeling for objects evolving in impulsive environnements. Wireless Communications and Networking Conference Workshops (WCNCW), 2012 IEEE, 2012, France. pp.191 - 195, 10.1109/GLOCOMW.2010.5700305 . hal-00732995

\section{HAL Id: hal-00732995 \\ https://hal.archives-ouvertes.fr/hal-00732995}

Submitted on 17 Sep 2012

HAL is a multi-disciplinary open access archive for the deposit and dissemination of scientific research documents, whether they are published or not. The documents may come from teaching and research institutions in France or abroad, or from public or private research centers.
L'archive ouverte pluridisciplinaire HAL, est destinée au dépôt et à la diffusion de documents scientifiques de niveau recherche, publiés ou non, émanant des établissements d'enseignement et de recherche français ou étrangers, des laboratoires publics ou privés. 


\title{
UWB channel modeling for objects evolving in impulsive environnements
}

\author{
Nourddine Azzaoui and Laurent Clavier, Member, IEEE
}

\begin{abstract}
We consider channel modeling issues in the context where communicating objects are evolving in impulsive environments. It was shown recently that $\alpha$-stable random processes are attractive solution for representing the ultra wide band communication channel in relatively large spatial areas. In this paper, we consider the $\alpha$-stable channel modeling in an evolutionary context where the model features depend on spatial locations. We introduce a methodological approach consisting of two parametric and non parametric components: the latter can be seen as black box model to describe the spatial evolution and it can be learned from historical observations of the transfer function. The other component concerns the frequency dependence and has an auto-regressive structure.
\end{abstract}

\section{INTRODUCTION}

One important difficulty of statistical channel modeling (especially when ultra wide band (UWB) is considered) resides in its ability to represent the time and environment evolutions. This channel variability is obvious when it comes to model mobiles and rapidly changing environments. In order to make realistic simulations, it is necessary to adapt the existing models to such situations. Indeed, statistical models, even those taking into account rare events ( $\alpha$-stable models), are not sufficient to describe the complexity of the channel behavior in all circumstances. Another challenge for developing real world applications is the fact that the specification of a such model needs a large measurement campaign and usually takes a lot of time to estimate the model parameters. For the next generation communication systems, (as WSN, inter vehicles, ...), time evolution can be slow or fast and nodes will change their position and interact with different communication infrastructures. They must be able to learn their environments autonomously, especially channel models of the medium in which they evolve. Due to the models complexity and possible fast evolution, this task is quasi impossible for the end user, especially low complexity sensor nodes.

The idea of this work is to give a general model that can adapt to the rapid change of the environment and learned from a limited number of measurements. For this purpose our proposed model must, from one hand, describe the space dependence of the channel transfer function and, on the other hand, the model variation with frequency. From this last point of view many works described this frequency dependence as

Nourddine Azzaoui is with the Mathematics Laboratory UMR-CNRS 6620 from of Blaise Pascal university, Aubière (France).

Laurent Clavier is with IEMN (Institute of Electronics, Microelectronics and Nanotechnology, UMR CNRS 8520), IRCICA (Research Institute on software and hardware components of the future for information and communication, FR CNRS 3024) and TELECOM Institute, TELECOM Lille 1, Lille, France. This work was supported by the ERDF (European Regional Development Fund) and by the Nord-Pas-De-Calais Region (France). parametric model: we cite for instance Ghassemzadeh [1] who uses a second-order autoregressive model $\mathbf{A R}(\mathbf{2})$ for frequency response generation of the UWB indoor channel. The work in this paper is an on going contribution, it presents the theory behind the model and solutions for space and frequency channel evolution in section I and some mathematical tools to estimate the parameters (section II) and validate the model (section III). Further investigations is needed to validate this approach. We also assume that the channel is accurately represented by an $\alpha$-stable model as we proposed in [2].

\section{THE EVOLUTIONARY CHANNEL MODEL}

The main idea of the evolutionary model comes from the fact that transfer functions measured at two contiguous places will be strongly dependent; it will be weakly dependent when the distance increases between two measurements. For the mathematical formulation let us denote $\mathcal{H}_{x}$ the transfer function at a position $x$. We postulate that the dependence will have a memory of length $p$. This space dependence can be formulated using conditional expectations as follows:

$$
\mathcal{H}_{x+1}=\mathbf{f}\left(\mathcal{H}_{x}, \ldots, \mathcal{H}_{x-p}\right)+\Upsilon
$$

where $\mathbf{f}$ is an unknown function not depending on the location and $\Upsilon=\mathcal{H}_{x+1}-\mathbb{E}\left(\mathcal{H}_{x+1} \mid \mathcal{H}_{x}, \ldots, \mathcal{H}_{x-p}\right)$. Equation (1) can be seen as a black-box model which does not support any $a$ priori about the environment. One of its main advantages is the fact that it can be learned (estimated non parametrically using kernel techniques) from historical observations collected by a given node. In order to understand the frequency dependence we present the model for observed transfer functions at $w_{1}, \ldots, w_{n}$, as the following:

$$
\text { 气. }\left\{\begin{array}{ccc}
\mathcal{H}_{p+1}\left(w_{1}\right) & = & \mathbf{f}\left(\mathcal{H}_{p}\left(w_{1}\right), \ldots, \mathcal{H}_{1}\left(w_{1}\right)\right)+\Upsilon_{p}\left(w_{1}\right) \\
\vdots & & \vdots \\
\mathcal{H}_{p+1}\left(w_{n}\right) & = & \mathbf{f}\left(\mathcal{H}_{p}\left(w_{n}\right), \ldots, \mathcal{H}_{1}\left(w_{n}\right)\right)+\Upsilon_{p}\left(w_{n}\right)
\end{array}\right.
$$

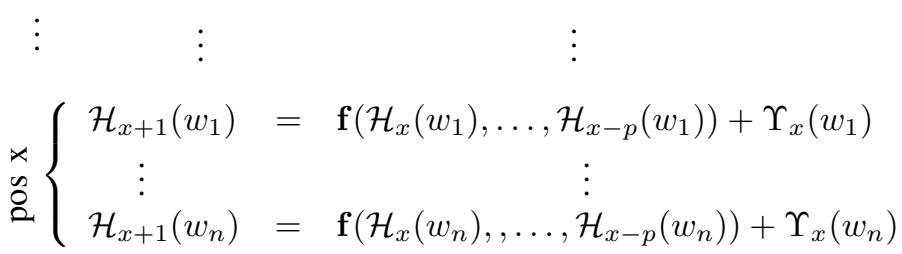

Index $p$ denotes the length of the dependence, consequently, the number of samples that nodes has to store in order to start its estimation. For different positions $x$ and $x^{\prime}$, we suppose that vectors $\Upsilon_{x}$ and $\Upsilon_{x^{\prime}}$ are pairwise independent. Since the function $f$ has captured all the intrinsic spatial influence, it makes more sense to suppose that $\Upsilon$ depends only on 
frequencies. Inspired from works in [1], we suppose that for a fixed position, the internal frequency dependence of $\Upsilon$ may be described by an autoregressive scheme.

$$
\Upsilon_{x}\left(w_{i}\right)=\sum_{k=1}^{q} \theta_{k} \Upsilon_{x}\left(w_{i-k}\right)+\varepsilon_{x, i}
$$

In order to take into account the impulsive nature and the $\alpha$-stable model [2], we suppose that errors $\varepsilon_{x, i}$ 's are i.i.d. symmetric $\alpha$-stable white noises.

The main difficulty in the black-box model (1) arises when we try to estimate the non parametric function $\mathbf{f}$ which will be seriously affected when $p$ becomes large; this is known as the curse of dimensionality. In order to overcome such an inconvenience we will suppose that, instead of $\left(\mathcal{H}_{x}, \ldots, \mathcal{H}_{x-p}\right)$, the transfer function $\mathcal{H}_{x+1}$ depends on a linear combination of this vector. This reduce $f$ to a real function and hence its estimation is facilitated. This will lead to the following:

$\mathcal{H}_{x+1}\left(w_{i}\right)=\mathbf{f}\left(\sum_{k=0}^{p} \eta_{k} \mathcal{H}_{x-k}\left(\omega_{i}\right)\right)+\sum_{k=1}^{q} \theta_{k} \Upsilon_{x}\left(w_{i-k}\right)+\varepsilon_{x, i}$

To resume, $f$ represents the space dependence, the coefficient $\theta_{k}$ the frequency linear dependence and $\varepsilon_{x, i}$ the unpredictable variations. This model is very general and will allow the use of powerful mathematical tools known in literature as semi parametric single index partially linear model. They have been largely studied in literature we cite among others [3], [4], [5], [6]... For compatibility with the semi-parametric formalism we rearrange the observed transfer functions as follows: we begin by taking $N=n m$ where $n$ is the number of positions and $m$ is the number of observed frequencies. For every $x=$ $1, \ldots, n-1$, for every $i=1, \ldots, m$ we take $s=(x-1) m+i$ and we denote:

$$
\begin{aligned}
& Y_{s}=\mathcal{H}_{x+1}\left(w_{i}\right), \\
& U_{s}=\left[\mathcal{H}_{x}\left(w_{i}\right), \ldots, \mathcal{H}_{x-p}\left(w_{i}\right)\right]^{\dagger}, \\
& V_{s}=\left[\Upsilon_{x}\left(w_{i-1}\right), \ldots, \Upsilon_{x}\left(w_{i-q}\right)\right]^{\dagger}, \\
& X_{s}=\left[U_{s}^{\dagger}, V_{s}^{\dagger}\right]^{\dagger}, \\
& \varepsilon_{s}=\varepsilon_{x, i}
\end{aligned}
$$

where $\dagger$ states for matrix transpose. This will lead to the conventional semi parametric single index model notations:

$$
Y_{s}=X_{s}^{\dagger} \boldsymbol{\theta}+\mathbf{f}\left(X_{s}^{\dagger} \boldsymbol{\eta}\right)+\varepsilon_{s}
$$

where $\boldsymbol{\theta}=\left(\mathbf{0}_{p+1}, \quad \theta_{1}, \ldots, \theta_{q}\right)$ and $\boldsymbol{\eta}=\left(\eta_{0}, \ldots, \eta_{p}, \quad \mathbf{0}_{q}\right)$ and $\mathbf{0}_{p}$ is zeroes vector in $\mathbb{R}^{p}$.

Motivation for using (4) for independent data analysis can be found in [5], [6], [7]. Many indications suggest the use of semi-parametric models (4); for example two transfer functions are presented in Fig. 1. Figures Fig. 2 and Fig. 3 illustrate the space and frequency dependence. The autoregressive scheme was also noticed in [1] and confort our proposal.

\section{STATISTICAL ESTIMATION AND MODEL SPECIFICATION FOR THE EVOLUTIONARY MODEL}

In this paper, we will address the estimation problem in the $\alpha$-stable case and introduce consistent estimators of unknown

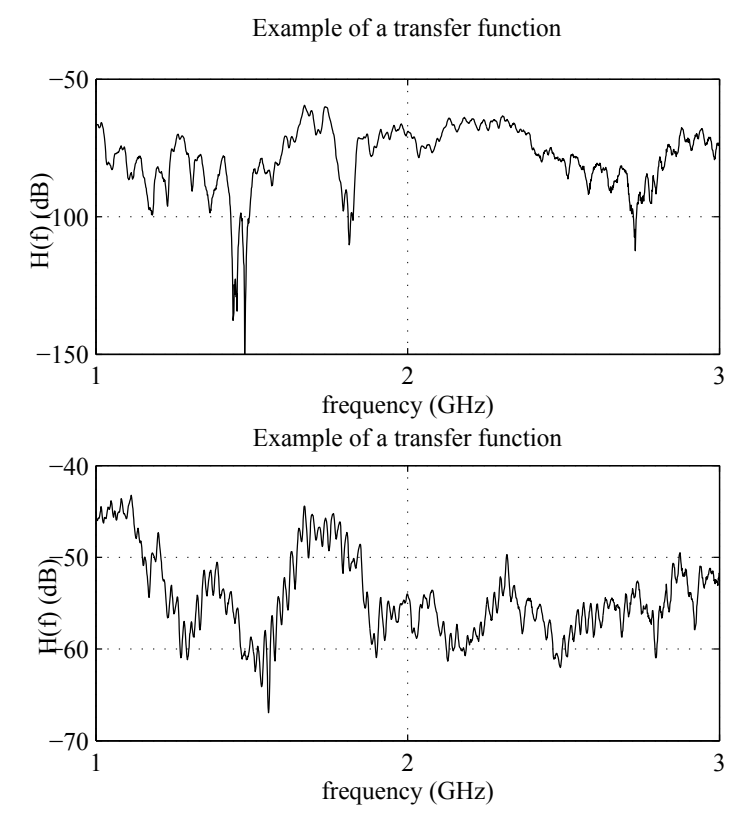

Fig. 1. Examples of measured transfer functions between $57-59 \mathrm{GHz}$ and down converted between $1-3 \mathrm{GHz}$ at two different locations. More details on the measurement setup can be found in [2], [8]

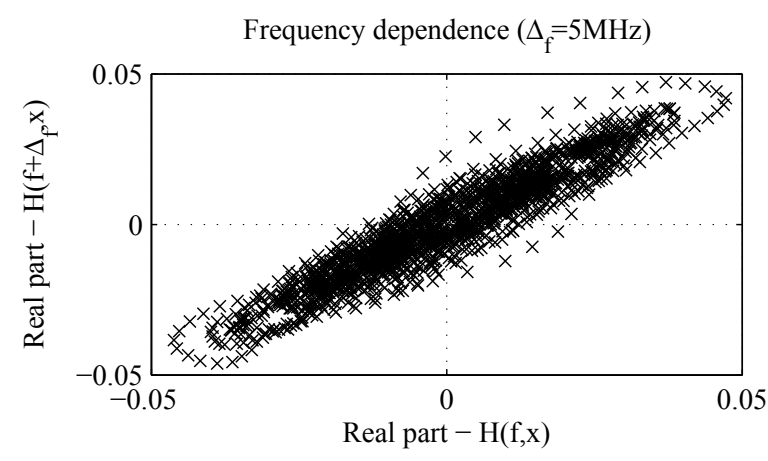

Frequency dependence $\left(\Delta_{\mathrm{f}}=5 \mathrm{MHz}\right)$

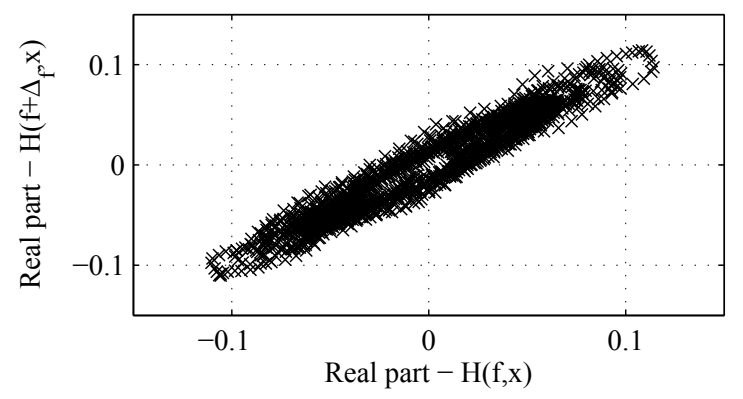

Fig. 2. Spatial dependence structure in observed transfer functions. The distance between $\mathrm{x}$ and $\mathrm{x}+1$ is $2.5 \mathrm{~mm}$. Two different locations in the computer room are considered. 

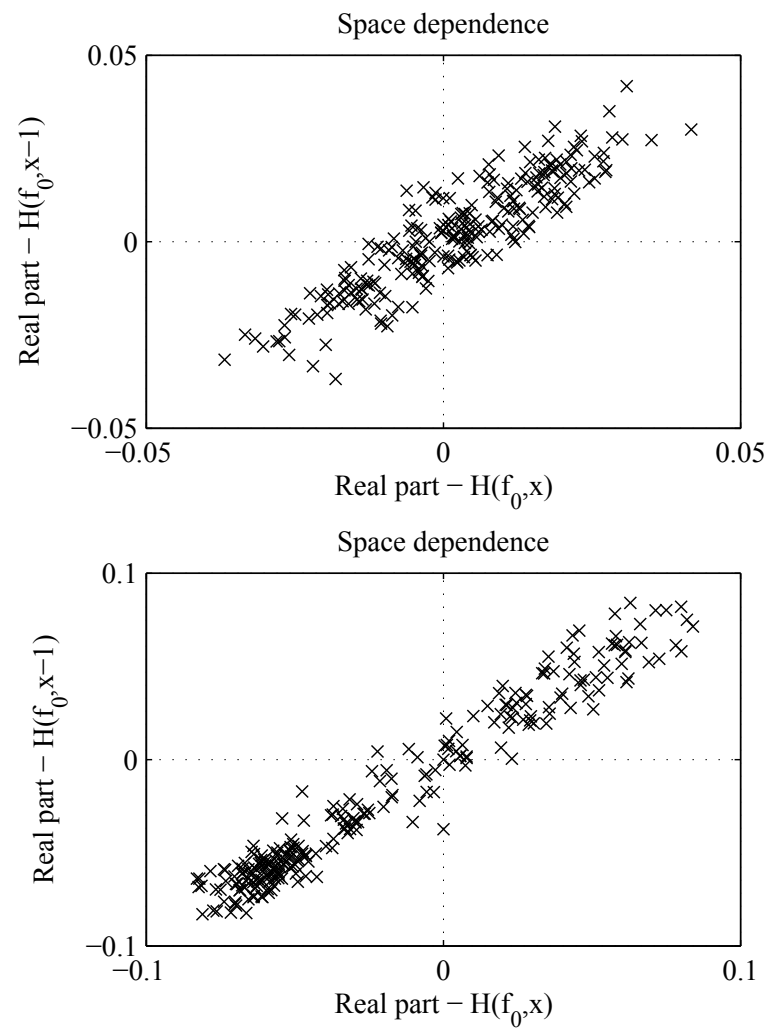

Fig. 3. Frequency dependence structure in observed transfer functions. The frequency step is $5 \mathrm{MHz}$.

model (4) features; the mode of convergence and the quality of the estimators will be detailed in further works.

\section{A. Estimation in semi parametric single index models}

Consider a semi-parametric single-index model of the form:

$$
Y_{s}=X_{s}^{\dagger} \boldsymbol{\theta}+\mathbf{f}\left(X_{s}^{\dagger} \boldsymbol{\eta}\right)+\varepsilon_{s}, s=1,2, \ldots N,
$$

where both $\boldsymbol{\theta}$ and $\boldsymbol{\eta}$ are vectors of unknown parameters. The real function $\mathbf{f}(\cdot)$ is unknown defined on $\mathbb{R}$ and is supposed to be twice differentiable. The random variables $\left\{\varepsilon_{s}\right\}$ are a sequence of errors with $\mathbb{E}\left[\varepsilon_{s} \mid X_{s}\right]=0$. A huge amount of works about such models have been introduced in literature especially when the errors are random variables with finite second order moments; for more details see for instance [5] and [7]. However no works have been done in the case of infinite variance errors. In this paper we suppose that the $\varepsilon_{s}$ 's are i.i.d. $\alpha$-stable centered variables with a fixed scale parameter $\sigma$.

In order to estimate the unknown parameters $\boldsymbol{\eta}, \boldsymbol{\theta}$ and the function $\mathbf{f}$ involved in (5), we introduce the following notation:

$$
\begin{gathered}
\mathbf{f}_{1 \boldsymbol{\eta}}(u)=\mathbb{E}\left[Y_{s} \mid X_{s}^{\dagger} \boldsymbol{\eta}=u\right], \\
\mathbf{f}_{2 \boldsymbol{\eta}}(u)=\mathbb{E}\left[X_{s} \mid X_{s}^{\dagger} \boldsymbol{\eta}=u\right],
\end{gathered}
$$

This decomposition is inspired from the conditional decomposition $\mathbf{f}(u)=\mathbb{E}\left[Y_{s}-X_{s}^{\dagger} \boldsymbol{\theta} \mid X_{s}^{\dagger} \boldsymbol{\eta}=u\right]$ which leads easily to the formula:

$$
\mathbf{f}(u)=\mathbf{f}_{1 \boldsymbol{\eta}}(u)-\left(\mathbf{f}_{2 \boldsymbol{\eta}}(u)\right)^{\dagger} \boldsymbol{\theta}
$$

Let $K$ be a kernel function i.e. a non negative even probability density (for example the gaussian kernel $K(x)=\frac{1}{\sqrt{2 \pi}} \mathrm{e}^{-\frac{x^{2}}{2}}$ ). From the conditional expectation given in (6) we propose a kernel estimation inspired from the Nadarya-Watson estimation techniques. We first estimate $\mathbf{f}_{1 \eta}(\cdot)$ by:

$$
\hat{\mathbf{f}}_{1 \boldsymbol{\eta}}(u)=\frac{\sum_{s=1}^{N} K_{h}\left(X_{s}^{\dagger} \boldsymbol{\eta}-u\right) Y_{s}}{\sum_{s=1}^{N} K_{h}\left(X_{s}^{\dagger} \boldsymbol{\eta}-u\right)}
$$

where $K_{h}(\cdot)=K(\dot{\bar{h}})$ and $h$ is a bandwidth parameter. Similarly we estimate the function $\mathbf{f}_{2 \eta}(\cdot)$ by:

$$
\hat{\mathbf{f}}_{2 \boldsymbol{\eta}}(u)=\frac{\sum_{s=1}^{N} K_{h}\left(X_{s}^{\dagger} \boldsymbol{\eta}-u\right) X_{s}}{\sum_{s=1}^{N} K_{h}\left(X_{s}^{\dagger} \boldsymbol{\eta}-u\right)}
$$

For a complete estimation of the function $\mathbf{f}$ we will need an estimate of the parameters $\boldsymbol{\eta}$ and $\boldsymbol{\theta}$. For this purpose we use the following notations:

$$
\begin{aligned}
& \bar{Y}_{s \boldsymbol{\eta}}=Y_{s}-\hat{\mathbf{f}}_{1 \boldsymbol{\eta}}\left(X_{s}^{\dagger} \boldsymbol{\eta}\right), \\
& \bar{X}_{s \boldsymbol{\eta}}=X_{s}-\hat{\mathbf{f}}_{2 \boldsymbol{\eta}}\left(X_{s}^{\dagger} \boldsymbol{\eta}\right),
\end{aligned}
$$

Let us consider the least-squares sum :

$$
S_{N}(\boldsymbol{\theta}, \boldsymbol{\eta} ; h)=\sum_{s=1}^{N}\left(\bar{Y}_{s \boldsymbol{\eta}}-\bar{X}_{s \boldsymbol{\eta}}^{\dagger} \boldsymbol{\theta}\right)^{2}
$$

The estimation idea consist in minimizing $S_{N}(\boldsymbol{\theta}, \boldsymbol{\eta}, h)$ over $(\boldsymbol{\theta}, \boldsymbol{\eta}, h)$. We first remark that, for a fixed $(\boldsymbol{\eta}, h)$, the least squares estimator of $\boldsymbol{\theta}$ can be deduced using classical linear regression techniques, it is given by:

$$
\hat{\boldsymbol{\theta}}(\boldsymbol{\eta}, h)=\left(\sum_{s=1}^{N} \bar{X}_{s \boldsymbol{\eta}} \bar{X}_{s \boldsymbol{\eta}}^{\dagger}\right)^{+} \sum_{s=1}^{N} \bar{X}_{s \boldsymbol{\eta}} \bar{Y}_{s \boldsymbol{\eta}},
$$

where $(.)^{+}$denotes matrix pseudo-inverse. We then estimate $(\boldsymbol{\eta}, h)$ by $(\hat{\boldsymbol{\eta}}, \hat{h})$ through minimizing,

$$
\hat{S}_{N}(\boldsymbol{\eta}, h)=\sum_{s=1}^{N}\left(\bar{Y}_{s \boldsymbol{\eta}}-\bar{X}_{s \boldsymbol{\eta}}^{\dagger} \hat{\boldsymbol{\theta}}(\boldsymbol{\eta}, h)\right)^{2}
$$

Inspired from the equation (8), we propose the nonparametric estimator of $\mathbf{f}(\cdot)$ by using estimates of $\mathbf{f}_{1 \boldsymbol{\eta}}$ and $\mathbf{f}_{2 \boldsymbol{\eta}}$ :

$$
\hat{\mathbf{f}}(u)=\hat{\mathbf{f}}_{1 \hat{\boldsymbol{\eta}}}(u)-\left(\hat{\mathbf{f}}_{2 \hat{\boldsymbol{\eta}}}(u)\right)^{\dagger} \hat{\boldsymbol{\theta}}(\hat{\boldsymbol{\eta}}, \hat{h}) .
$$

On the other hand, when the errors scale parameter $\sigma$ is unknown, it can be estimated using the fractional lower moments technique as follows:

$$
\hat{\sigma}=C_{\alpha}(\rho)\left(\frac{1}{N} \sum_{s=1}^{N}\left|\bar{Y}_{s \hat{\boldsymbol{\eta}}}-\bar{X}_{s \hat{\boldsymbol{\eta}}}^{\dagger} \hat{\boldsymbol{\theta}}(\hat{\boldsymbol{\eta}}, \hat{h})\right|^{\rho}\right)^{\frac{1}{\rho}}
$$

for every $1<\rho<\alpha$ and $C_{\alpha}(\rho)$ is an universal constant depending only on $\alpha$ and $\rho$, it is given by:

$$
C_{\alpha}(\rho)=\left(\frac{\alpha \sqrt{\pi} \Gamma\left(\frac{-\rho}{2}\right)}{2^{\rho+1} \Gamma\left(\frac{1+\rho}{2}\right) \Gamma\left(\frac{-\rho}{\alpha}\right)}\right)^{\frac{1}{\rho}}
$$


The mode of convergence and the consistency of these estimators was studied in the second order case, the reader find a detailed overview in literature; see for instance [7]. In this last work a central limit theorem type result was established for all estimators. We believe that similar results may be proven for the $\alpha$-stable process and will be similar to the generalized central limit theorem context.

\section{MODELS ADEQUACY AND HYPOTHESIS TESTING}

In this section we present statistical techniques to test the adequacy of semi parametric models of type (4). Recently, semiparametric approach has been used for model specification tests in the case of finite variance processes. We will proceed by analogy to second order works, we focus on partially linear or single-index scheme against a general non-parametric form. We concentrate on parametric specification testing of the conditional mean function defined for $u \in \mathbb{R}^{p+q+1}$ by:

$$
\left.m(u)=\mathbb{E}\left[Y_{s} \mid X_{s}=u\right)\right]
$$

\section{A. Testing for single-index regression}

In this subsection we focus on the particular case of (4) without the linear regression component. The test purpose is to see if transfer functions evolutions can be reduced to the space evolution, not considering the frequency dependency $\left(\mathbb{H}_{0}\right)$. Somehow it can be seen as a test for spatial stationarity of transfer functions. We thus look at testing the null hypothesis of single index modeling against a class of non parametric functions:

$$
\begin{array}{ll}
\left(\mathbb{H}_{0}\right): & m(x)=\mathbf{f}\left(x^{\dagger} \boldsymbol{\eta}\right), \\
\left(\mathbb{H}_{1}\right): & m(x)=\mathbf{f}\left(x^{\dagger} \boldsymbol{\eta}\right)+\Delta(x) \text { for all } x \in \mathbb{R}^{p+1},
\end{array}
$$

where $\mathbf{f}(\cdot)$ is an unknown function on $\mathbb{R}, \boldsymbol{\eta}$ is a vector of unknown parameters and $\Delta$ is any regular function defined on $\mathbb{R}^{p+1}$. Under the null hypothesis $\left(\mathbb{H}_{0}\right)$ we have given techniques to estimate $\boldsymbol{\eta}$ in section II. In the single index particular case the estimate of $\mathbf{f}(\cdot)$ will be simplified and is given by:

$$
\hat{\mathbf{f}}\left(X_{s}^{\dagger} \boldsymbol{\eta}\right)=\frac{\sum_{t=1}^{N} Y_{t} K\left(\frac{\left(X_{s}-X_{t}\right)^{\dagger} \boldsymbol{\eta}}{h}\right)}{\sum_{u=1}^{N} K\left(\frac{\left(X_{s}-X_{u}\right)^{\dagger} \boldsymbol{\eta}}{h}\right)},
$$

with $K(\cdot)$ being a kernel function defined on $\mathbb{R}$. Consequently, from (10) the parameter $\boldsymbol{\eta}$ is then estimated by minimizing:

$$
\hat{\boldsymbol{\eta}}=\arg \min _{(\boldsymbol{\eta}, h)} \sum_{s=1}^{N}\left(Y_{s}-\hat{\mathbf{f}}\left(X_{s}^{\dagger} \boldsymbol{\eta}\right)\right)^{2}
$$

As we have supposed that the errors $\varepsilon_{s}$ 's are $\alpha$-stable i.i.d. centered random variables, then by the generalized central limit theorem it is more convenient to approximate the estimated errors $\hat{Y}_{s}=Y_{s}-\hat{\mathbf{f}}\left(X_{s}^{\dagger} \hat{\boldsymbol{\eta}}\right)$ by a centered symmetric $\alpha$-stable distribution with estimated scale parameter $\hat{\sigma}$ :

$$
\hat{\sigma}=C_{\alpha}(\rho)\left(\frac{1}{N} \sum_{s=1}^{N}\left|\hat{Y}_{s}\right|^{\rho}\right)^{\frac{1}{\rho}}
$$

By analogy with the second order case and for compatibility with infinite variance we suggest the test statistic:

$$
\mathcal{L}=\frac{\sum_{s=1}^{N}\left|\hat{Y}_{s}\right|}{\hat{\sigma}}
$$

Many improvements are still needed in the case of stable variables, including the asymptotic distribution and robustness of the statistic $\mathcal{L}$. For the hypotheses testing in the case of second order semi parametric models one can find a rich literature in [9], [4], [10] and references within.

\section{B. Testing for partially linear single-index model}

It is a natural extension of the single index model to situations where the linear regression component may be suitable for modeling the inherent studied phenomenon. The following test evaluates if the dependency on space and frequency is sufficient $\left(\mathbb{H}_{0}\right)$ or if it would be more accurate to take some more phenomena into account, for instance some non linear dependency in frequency.

$$
\begin{array}{ll}
\left(\mathbb{H}_{0}\right): & m(x)=x^{\dagger} \boldsymbol{\theta}+\mathbf{f}\left(x^{\dagger} \boldsymbol{\eta}\right) \\
\left(\mathbb{H}_{1}\right): & m(x)=x^{\dagger} \boldsymbol{\theta}+\mathbf{f}\left(x^{\dagger} \boldsymbol{\eta}\right)+\Delta(x),
\end{array}
$$

for all $x \in \mathbb{R}^{p+q+1}$. This test problem has been studied for the second order case by [5], [6]. With the same reasoning as in the single index model and by using the estimation techniques under the hypothesis $\left(\mathbb{H}_{0}\right)$ presented in section II, we propose the test statistic:

$$
\mathcal{L}=\frac{\sum_{s=1}^{N}\left|\widehat{\bar{Y}}_{s}\right|}{\hat{\sigma}}
$$

where $\widehat{\bar{Y}_{s}}=Y_{s}-X_{s}^{\dagger} \hat{\boldsymbol{\theta}}-\hat{\mathbf{f}}\left(X_{s}^{\dagger} \hat{\boldsymbol{\eta}}\right)$, the quantities $\hat{\boldsymbol{\theta}}, \hat{\boldsymbol{\eta}}, \hat{\mathbf{f}}(\cdot)$ and $\hat{\sigma}$ are respectively the consistent estimators given in (9), (10), (11) and (12). One of the main issues to evaluate the models compatibility is to examine the power and the size tests :

$$
\begin{aligned}
\alpha & =\mathbb{P}\left(\mathcal{L}>l_{\alpha} \mid \text { such that } \mathbb{H}_{0} \text { "holds" }\right) \\
\beta & =\mathbb{P}\left(\mathcal{L}>l_{\alpha} \mid \text { such that } \mathbb{H}_{1} \text { "holds" }\right)
\end{aligned}
$$

where $l_{\alpha}$ will be tabulated from the distribution of $\mathcal{L}$. It should be noted that the estimator presented here must be extensively studied for asymptotic properties and robustness.

\section{CONCLUSION AND PERSPECTIVES}

In this paper we have introduced a methodological approach that adapts semi-parametric techniques to the context of spatial ultra wide band channel modeling. Our approach may be seen as a general-purpose framework for the modeling of the spatial evolution of the channel transfer functions described by an $\alpha$ stable random process. Extensive simulations on real data must be performed to evaluate the adequacy of the proposed model in the context of indoor and outdoor configurations and this paper only presents the general framework. 


\section{REFERENCES}

[1] S. Ghassemzadeh, R. Jana, C. Rice, W. Turin, and V. Tarokh, "Measurement and modeling of an ultra-wide bandwidth indoor channel," IEEE Transactions on Communications, vol. 52, no. 10, pp. 1786-1796, 2004.

[2] N. Azzaoui and L. Clavier, "Statistical channel model based on $\alpha$ stable random processes and application to the $60 \mathrm{GHz}$ ultra wide band channel," IEEE Transactions on Communications, vol. 58, no. 5, pp. 1457-1467, 2010.

[3] R. Carroll, J. Fan, I. Gijbels, and M. Wand, "Generalized partially linear single-index models," Journal of the American Statistical Association, vol. 92, no. 438, pp. 477-489, 1997.

[4] J. Gao and H. Liang, "Statistical inference in single-index and partially nonlinear models," Annals of the Institute of Statistical Mathematics, vol. 49, no. 3, pp. 493-517, 1997.

[5] Y. Xia, H. Tong, and W. Li, "On extended partially linear single-index models," Biometrika, vol. 86, no. 4, p. 831, 1999.

[6] Y. Xia, W. Li, H. Tong, and D. Zhang, "A goodness-of-fit test for singleindex models," Statistica Sinica, vol. 14, no. 1, pp. 1-27, 2004.

[7] J. Gao, Nonlinear Time Series Semiparametric and Nonparametric Methods. Chapman \& Hall/CRC, 2007.

[8] M. Fryziel, C. Loyez, L. Clavier, and R. N., "Path loss model of the 60 GHz radio channel," Microwave and optical technology letters, vol. 34, no. 3, pp. 158-162, Aug. 2002.

[9] Y. Fan and Q. Li, "Consistent model specification tests: omitted variables and semiparametric functional forms," Econometrica: Journal of the Econometric Society, vol. 64, no. 4, pp. 865-890, 1996.

[10] W. Stute and L. Zhu, "Nonparametric checks for single-index models," Annals of Statistics, vol. 33, no. 3, pp. 1048-1083, 2005. 